\title{
The Jacobl-Perron algorithm and the algebra of recursive sequences
}

\section{A.G. Shannon and Leon Bernsteln}

\begin{abstract}
This paper shows the existence of a one-to-one correspondence between a certain class of square matrices of arbitrary order and a related extension field. The elements of these matrices are obtained from certain basic linear recursive sequences by means of a generalization of the euclidean algorithm.
\end{abstract}

\section{Introduction}

Ward [6] established the existence of a one-to-one correspondence between a certain class of square matrices of order three (with elements from the field $F$ of the coefficients of the auxiliary polynomial $F(x)$ of a third order recurrence relation) and the field $F(\alpha)$ formed by the adjunction to $F$ of a root $\alpha$ of $F(x)=0$. It is assumed throughout the paper that $F(x)$ is irreducible in $F$.

In this paper Ward's results are generalized to sequences of arbitrary order. The elements of the class of square matrices of arbitrary order are related to certain basic linear recursive sequences by means of a JacobiPerron algorithm for these elements.

Jacobi-Perron algorithms are generalizations of the euclidean algorithm and a definition is formulated in Section 5. This particular algorithm is shown to be periodic, which fact means that the central result can also be proved by the extensive theory developed in recent years by Bernstein [2, and elsewhere], to whom the name of the algorithm is due.

Received 21 November 1972. 


\section{Basic definitions}

We define $r$ "basic" sequences of order $r,\left\{U_{s n}^{(r)}\right\}$, $s=1,2, \ldots, r$, by the recurrence relation

$$
U_{s, n+r}^{(r)}=\sum_{j=1}^{r}(-1)^{j+1} P_{r, j} U_{s, n+r-j}^{(r)}(n>r)
$$

and the initial terms

$$
U_{s, n}^{(r)}=\delta_{s, n}
$$

(the Kronecker delta), where the $P_{r j}$ are arbitrary integers. The "primordial" sequence of order $r,\left\{U_{0 n}^{(r)}\right\}$ also satisfies this recurrence relation, but has initial terms given by

$$
\begin{aligned}
& U_{0, n}^{(r)}=0, n<1, \\
& U_{0, n}^{(r)}=\sum_{j=1}^{r} \alpha_{r, j}^{n-1}, \quad 1 \leq n \leq r .,
\end{aligned}
$$

where the $\alpha_{r, j}$ are the roots of the auxiliary equation associated with the recurrence relation (2.1).

The first result of interest is

$$
v_{0, n}^{(r)}=\sum_{j=1}^{r} v_{j, n+j-1}^{(r)}
$$

The proof of this follows from (3.1) and Theorem 1. below.

\section{Matrices}

Let $M_{n}^{(r)}$ denote the square matrix of order $r$ 
(3.1)

$$
M_{n}^{(r)}=\left[\begin{array}{cccc}
U_{1, n+1}^{(r)} & U_{2, n+1}^{(r)} & \cdots & U_{r, n+1}^{(r)} \\
U_{1, n+2}^{(r)} & U_{2, n+2}^{(r)} & \cdots & U_{r, n+2}^{(r)} \\
& \cdots & & \\
U_{1, n+r}^{(r)} & U_{2, n+r}^{(r)} & \cdots & U_{r, n+r}^{(r)}
\end{array}\right]
$$

Thus $M_{0}^{(r)}=I_{r}$, the unit matrix of order $r$, and

$$
M_{1}^{(r)}=\left[\begin{array}{ccccc}
0 & 1 & 0 & \ldots & 0 \\
0 & 0 & 1 & \ldots & 0 \\
0 & 0 & 0 & \ldots & 1 \\
(-1)^{n+1} P_{r, r} & \ldots & \ldots & P_{r, 1}
\end{array}\right] \equiv M,
$$

for convenience. Properties (3.2) to (3.5) are easily proved by induction:

$$
\begin{aligned}
M_{n+1}^{(r)} & =M_{n}^{(r)}=M^{n+1}, \\
M_{n}^{(r)} M_{m}^{(r)} & =M_{m}^{(r)} M_{n}^{(r)}=M_{m+n}^{(r)}, \\
M_{n+r}^{(r)} & =\sum_{j=1}^{r}(-1)^{j+1} P_{r, j} M_{n+r-j}^{(r)}, \\
M_{n-1}^{(r)} & =\sum_{j=1}^{r} U_{j, n}^{(r)} M_{j-1}^{(r)}, \quad 1 \leq n \leq r .
\end{aligned}
$$

(3.2) shows that $M_{n}^{(r)}$ is a particular solution of the matrix difference equation of order one

$$
\Omega_{n+1}=M \Omega_{n}
$$

and (3.4) shows that $M_{n}^{(r)}$ is a particular solution of

$$
\Omega_{n}=\sum_{j=1}^{r}(-1)^{j+1} P_{r, j} \Omega_{n-j} .
$$

THEOREM 1. The characteristic function of the matrix $M_{n}^{(r)}$ is 


$$
\lambda^{r}-\sum_{m=1}^{r}(-1)^{m+1} s(r, n, m) \lambda^{r-m},
$$

where $\varepsilon(r, n, m)$ is the symmetric function of the roots $\alpha_{r j}$ of the auxiliary equation of (2.1) taken $m$ at a time:

$$
\varepsilon(r, n, m)=\left[\alpha_{r, j_{1}}^{n} \alpha_{r, j_{2}}^{n} \cdots \alpha_{r, j_{m}}^{n} .\right.
$$

Proof. Suppose that the roots, $\alpha_{r j}$, of the auxiliary equation associated with the recurrence relation (2.1) are distinct, where

$$
F(x) \equiv \prod_{j=1}^{r}\left(x-\alpha_{r j j}\right)=0 .
$$

Then the matrix $M$ has $r$ linearly independent eigenvectors; that is, there are $r$ distinct roots of the auxiliary equation

$$
0=\operatorname{det}(M-\lambda I) \text {. }
$$

This follows because

$$
\begin{aligned}
\operatorname{det}(\lambda I-M) & =\left|\begin{array}{rrrrr}
\lambda & -1 & 0 & \ldots & 0 \\
0 & \lambda & -1 & \ldots & 0 \\
0 & 0 & 0 & & -1 \\
(-1)^{r}{ }_{r, r} & \cdots & \ldots & \lambda-P_{r, 1}
\end{array}\right| \\
& =\lambda\left(\lambda^{r-1}-P_{r, 1} \lambda^{r-2}+\ldots\right)+(-1)^{r_{P}} r, r \\
& =\lambda^{r}-\sum_{j=1}^{r}(-1)^{j+1} P_{r, j} \lambda^{r-j} \\
& =F(\lambda),
\end{aligned}
$$

so that the eigenvalues of $M$ are the roots, $\alpha_{r, j}$, of the auxiliary equation associated with the recurrence relation. Thus there exists a nonsingular matrix $X$ :

$$
M^{*}=\operatorname{diag}\left(\alpha_{r, 1}, \alpha_{r, 2}, \ldots, \alpha_{r, 2}\right)=X^{-1} M X
$$




$$
\begin{aligned}
M^{*^{2}} & \equiv\left(M^{*}\right)^{2} \\
& =\operatorname{diag}\left(\alpha_{r, 1}^{2}, \alpha_{r, 2}^{2}, \ldots, \alpha_{r, r}^{2}\right) \\
& =X^{-1} M^{2} X \\
& =X^{-1} M_{2}^{(r)} X
\end{aligned}
$$

from (3.2). Similarly,

$$
\begin{aligned}
M^{*^{n}} & =\operatorname{diag}\left(\alpha_{r, 1}^{n}, \alpha_{r, 2}^{n}, \ldots, \alpha_{r, r}^{n}\right) \\
& =X^{-1} M_{n}^{(r)} X,
\end{aligned}
$$

which implies that the eigenvalues of $M_{n}^{(r)}$ are $\alpha_{r, j}^{n}(j=1, \ldots, r)$. The result then follows from the definition of the characteristic polynomial of the matrix $M_{n}^{(r)}$.

\section{THEOREM 2.}

$$
\boldsymbol{s}(r, n, m)=\sum(-1)^{n m+\Sigma \lambda} \frac{(n m-1) ! \Sigma \lambda}{\lambda_{1} ! \lambda_{2} ! \ldots} \prod P_{r, j}^{\lambda_{j}}
$$

in which $\Sigma j \lambda_{j}=n m$.

Proof. For ease of notation we write

$$
s_{m}=\sum_{j=1}^{r} \alpha_{r, j}^{m} \quad\left(=U_{0, m+1}^{(r)}\right),
$$

and we express $s(r, n, m)$ in terms of ${ }_{m}^{s_{m}}$.

$$
\log \left\{\left(1-\alpha_{r, 1}^{n} x\right)\left(1-\alpha_{r, 2}^{n} x\right) \ldots\right\}=-\sum_{j=1}^{r}\left(\alpha_{r, j}^{n} x+\frac{1}{2} \alpha_{r, j}^{2 n} x^{2}+\frac{1}{3} \alpha_{r, j}^{3 n} x^{3}+\ldots\right)
$$

or

$$
\operatorname{Iog}\left(1-8(r, n, 1) x+8(x, n, 2) x^{2}-\ldots\right)=-8 n^{x-\frac{1}{2} 8} 2 n^{x^{2}}-\frac{1}{3} 83 n^{x^{3}}-\ldots
$$

or

$$
1-\sum_{m=1}^{\infty}(-1)^{m+1}{ }_{8}(r, n, m) x^{m}=\exp \left(-\sum_{m=1}^{\infty} \boldsymbol{s}_{m m} x^{m} / m n\right) \text {. }
$$


Expansion of the right-hand side, the use of the multinomial theorem and comparison of coefficients yield

$$
s(r, n, m)=\sum(-1)^{\sum(j+1) \lambda} j \frac{s_{n}^{\lambda_{1}} s_{2 n}^{\lambda_{2} s_{3}} \lambda_{3} \ldots}{1^{\lambda_{1} 2^{\lambda_{2}} 3^{\lambda_{3}} \ldots \lambda_{1} ! \lambda_{2} ! \lambda_{3} ! \ldots},}
$$

in which $m=\Sigma j \lambda_{j}$.

Now. MacMahon [5] has shown that

$$
P_{r, m}=\sum(-1)^{\sum(j+1) \lambda_{j}} \frac{s_{1}^{\lambda_{1} s_{2}^{\lambda_{2}} s_{3}^{\lambda_{3}} \ldots}}{1^{\lambda_{1}{ }^{\lambda_{2}} 3^{\lambda_{3}} \ldots \lambda_{1} ! \lambda_{2} ! \lambda_{3} !}},
$$

and

$$
s_{m}=\sum(-1)^{m+\Sigma \lambda} \frac{(m-1) ! \Sigma \lambda}{\lambda_{1} ! \lambda_{2} ! \ldots} \Pi P_{r, j}^{\lambda j}
$$

in which $\Sigma j \lambda_{j}=m$, from which the required result follows.

THEOREM 3. Suppose the eigenvalues of $M$ can be ordered so that $\alpha_{r, 1}$ corresponds to the eigenvalue of maximon modulus:

$\left|\alpha_{r, 1}\right|>\left|\alpha_{r, 2}\right| \geq\left|\alpha_{r, 3}\right| \geq \ldots \geq\left|\alpha_{r, r}\right|$, then

$$
\lim _{n \rightarrow \infty} U_{r, n+1}^{(r)} / U_{r, n}^{(r)}=\alpha_{r, 1} \text {. }
$$

Proof. The eigenvectors $x_{j}^{(r)}(j=1, \ldots, r)$ are linearly independent because the $\alpha_{r, j}$ are distinct. So we can find constants $b_{i, j} \quad\left(b_{1, j} \neq 0\right):$

$$
\mathrm{q}_{0, j}^{(r)}=\sum_{i=1}^{r} b_{i, j} \mathrm{x}_{i}^{(r)}
$$

where

$$
q_{n, j}^{(r)}=\left[U_{r, n+j}^{(r)}, U_{r, n+j+1}^{(r)}, \ldots, U_{r, n+j+r-1}^{(r)}\right]^{T}
$$

Set

$$
Q_{n}^{(r)}=\left[q_{n, 1}^{(r)}, q_{n, 2}^{(r)}, \ldots, q_{n, r}^{(r)}\right]
$$


so that

$$
\begin{aligned}
Q_{n}^{(r)} & =M Q_{n-1}^{(r)} \\
& =M^{2} Q_{n-2}^{(r)} \\
& =M^{n} Q_{0}^{r}
\end{aligned}
$$

and

$$
\begin{aligned}
\mathrm{q}_{n, j}^{(r)} & =M^{n} \mathrm{q}_{0, j}^{(r)} \\
& =\sum_{i=1}^{r} b_{i, j} \alpha_{r, i}^{n} x_{i},
\end{aligned}
$$

since the $\alpha_{r, i}$ are the eigenvalues of $M$.

$$
\text { If } \begin{aligned}
& \mathrm{x}_{i}= {\left[x_{1 i}, x_{2 i}, \ldots, x_{r i}\right]^{T} \text {, then } } \\
& \frac{U_{r, n+j+k}^{(r)}}{U_{r, n+j+k-1}^{(r)}}=\alpha_{r, 1} \frac{b_{1, j^{x} x_{1, k}}+\sum_{i=2}^{r} b_{i, j}\left(\alpha_{r, i} / \alpha_{r, 1}\right)^{n+1} x_{i, k}}{b_{1, j} x_{1, k}+\sum_{i=2}^{r} b_{i, j}\left(\alpha_{r, i} / \alpha_{r, 1}\right)^{n} x_{i, k}} \\
& \rightarrow \alpha_{r, 1} \text { as } n \rightarrow \infty,
\end{aligned}
$$

since $\left|\alpha_{r, i} / \alpha_{r, 1}\right|<1$ for $i=2, \ldots, r$. In particular,

$$
\lim _{n \rightarrow \infty} U_{r, n+1}^{(r)} / U_{r, n}^{(r)}=\alpha_{r, 1} \text {. }
$$

THEOREM 4. $\operatorname{det} Q_{n}^{(r)}=(-1)^{r(r-I) / 2} E_{r p}^{n}$ where

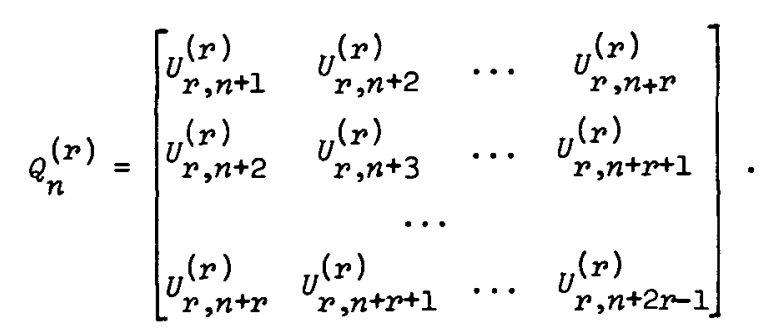

Proof. We use induction on $n$. 


$$
\begin{aligned}
& \operatorname{det} Q_{0}^{(r)}=\left|\begin{array}{cccc}
U_{r, 1}^{(r)} & U_{r, 2}^{(r)} & \cdots & U_{r, r}^{(r)} \\
U_{r, 2}^{(r)} & U_{r, 3}^{(r)} & \cdots & U_{r, r+1}^{(r)} \\
& & \cdots & \\
U_{r, r}^{(r)} & U_{r, r+1}^{(r)} & \cdots & U_{r, 2 r-1}^{(r)}
\end{array}\right| \\
& =\left|\begin{array}{cccc}
0 & 0 & \cdots & 1 \\
0 & 0 & \cdots & U_{r, r+1}^{(r)} \\
& \ldots & \\
1 & U_{r, r+1}^{(r)} & \cdots & U_{r, 2 r-1}^{(r)}
\end{array}\right| \\
& =(-1)^{r(r-1) / 2}, r \geq 2 \text {. } \\
& \operatorname{det}_{n}^{(r)}=\left|\begin{array}{ccc}
U_{r, n+1}^{(r)} & \cdots & (-1)^{r+1} P_{r, r} U_{r, n}^{(r)}+\sum_{j=1}^{r-1}(-1)^{j+1} P_{r, j} U_{r, n+r-j}^{(r)} \\
U_{r, n+2}^{(r)} & \cdots & (-1)^{r+1} P_{r, r} U_{r, n+1}^{(r)}+\sum_{j=1}^{r-1}(-1)^{j+1} P_{r, j} U_{r, n+r-j+1}^{(r)} \\
\cdots & (-1)^{r+1} P_{r, r} U_{r, n+r-1}^{(r)}+\sum_{j=1}^{r-1}(-1)^{j+1} P_{r, j} U_{r, n+2 r-j-1}^{(r)}
\end{array}\right| \\
& =(-1)^{r+1} P_{r, \dot{r}}\left|\begin{array}{cccc}
U_{r, n+1}^{(r)} & U_{r, n+2}^{(r)} & \cdots & U_{r, n}^{(r)} \\
U_{r, n+2}^{(r)} & U_{r, n+3}^{(r)} & \cdots & U_{r, n+1}^{(r)} \\
& \ldots & & \\
U_{r, n+r}^{(r)} & U_{r, n+r+1}^{(r)} & \cdots & U_{r, n+r-1}^{(r)}
\end{array}\right| \\
& =(-1)^{r-1}(-1)^{r+1} P_{r, r}\left|\begin{array}{ccc}
U_{r, n}^{(r)} & U_{r, n+1}^{(r)} & \cdots \\
U_{r, n+1}^{(r)} & U_{r, n+2}^{(r)} & \cdots \\
& \cdots & \\
U_{r, n+r-1}^{(r)} & U_{r, n+r}^{(r)} & \cdots
\end{array}\right| \\
& =(-1)^{2 r} P_{r, r} \operatorname{det}_{n-1}^{(r)} \text {. }
\end{aligned}
$$




$$
\begin{aligned}
& \operatorname{det} Q_{n}^{(r)}=P_{r, r} \operatorname{det} Q_{n-1}^{(r)} \\
& =P_{r, r^{\operatorname{det}} Q_{n-2}^{(r)}}^{2} \\
& =P_{r, r}^{n} \operatorname{det} Q_{0}^{(r)} \\
& =(-1)^{r(r-1) / 2} p_{r, r}^{n} \text {, }
\end{aligned}
$$

as required.

THEOREM 5. A vector solution of the Zinear diophantine equation

$$
U_{r, n+1}^{(r)} x_{1}+U_{r, n+2}^{(r)} x_{2}+\ldots+U_{r, n+r}^{(r)} x_{r}=1
$$

is given by the formula

$$
x_{m}=(-1)^{m+1+r(p-1) / 2} P_{r, r}^{-n}{ }_{m, r}(m=1,2, \ldots, r),
$$

where the $B_{m, x}$ are the cofactors of the elements in the $m$ th rows of the determinant $Q_{n}^{(r)}$.

Proof.

$$
\begin{aligned}
\sum_{m=1}^{r} U_{r, n+m}^{(r)}(-1)^{m+1} B_{m, r} & =\operatorname{det} Q_{n}^{(r)} \\
& =(-1)^{r(x-1) / 2} P_{r, r}^{n} .
\end{aligned}
$$

Thus

and so

$$
\sum_{m=1}^{r} U_{r, n+m}^{(r)}(-1)^{m+1+r(r-1) 2} P_{r, r^{B}{ }^{-n}, r}=1,
$$

$$
\begin{aligned}
x_{m} & =(-1)^{m+1+r(r-1) / 2} P_{r, r_{m, r}}^{-n} \\
\text { satisfies } \sum_{m=1}^{r} U_{r, n+m}^{(r)} x_{m} & =1 .
\end{aligned}
$$

4. Fields

The most general solution of $(3.6)$ is 


$$
\Omega_{n}=M_{n}^{(r)} \Omega_{0}
$$

where the elements of the matrix $\Omega_{0}$ are arbitrary. Let

$$
\Omega_{n}=R_{n}
$$

be a particular solution obtained by putting

$$
\Omega_{0}=R_{0}=\left[\begin{array}{cccc}
w_{0,0} & w_{1,0} & \ldots & w_{p-1,0} \\
w_{0,1} & w_{1,1} & \ldots & w_{p-1,1} \\
& \ldots & \\
w_{0, p-1} & w_{1, p-1} & \ldots & w_{p-1, p-1}
\end{array}\right],
$$

where $W_{0,0}, \ldots, W_{p-1, p-1}$ are elements of $F$. Then from (3.3) and $(3.4)$,

$$
\begin{gathered}
\text { (4.2) } \\
R_{m+n}=M_{n}^{(r)} R_{m}, \\
R_{n+r}=\sum_{j=1}^{n}(-1)^{j+1} P_{r, j} R_{n+r-j} .
\end{gathered}
$$

From (4.2) and (4.3) we obtain:

THEOREM 6. The sequence of matrices $\left\{R_{n}\right\}$ is a particular solution of the matrix difference equation (3.6), where the value of $R_{0}$ is given by (4.I), if and only if

$$
R_{n}=\left[\begin{array}{crrr}
W_{0, n} & W_{1, n} & \cdots & W_{p-1, n} \\
W_{0, n+1} & W_{1, n+1} & \cdots & W_{r-1, n+1} \\
W_{0, n+r-1} & W_{1, n+p-1} & \ldots & W_{r-1, n+r-1}
\end{array}\right],
$$

where the initial values of $\left\{w_{s, n}\right\},(s=0,1, \ldots, k-1)$, are given by $w_{s, 0}, \ldots, w_{s, r-1}$.

We shall now establish an isomorphism between $F(\alpha)$ and a certain class of matrix with elements in $F$.

THEOREM 7. The class $M$ of all matrices of the form 


$$
R=\sum_{j=0}^{n-1} w_{j} M^{j},
$$

where $w_{0}, W_{1}, \ldots, W_{p-1}$ are any elements of $F$, forms a field which is simply isomorphic with the field $F(\alpha)$ obtained by adjoining a root $\alpha$ of $F(x)=0$ to $F$.

Proof. It is clear from (3.1) and (3.2) that any matrix $R$ of $M$ can vanish when and only when $w_{0}, w_{1}, \ldots, w_{p-1}$ vanish.

$M$ is obviously closed under addition and subtraction; by (3.4) and $(3.2)$

$$
M^{r}=\sum_{j=1}^{r}(-1)^{j+1} P_{r, j} M^{p-j},
$$

so that $M$ is closed under multiplication. Furthermore, multiplication is commutative, and distributive with respect to addition.

Any element $\rho$ of the field $F(\alpha)$ may be put in the unique canonical form

$$
\rho=\sum_{j=0}^{n-1} w_{j} \alpha^{j},
$$

where the $W_{j}$ are elements of $F$. Set $M$ and $F(\alpha)$ into one-to-one correspondence by pairing the elements $\rho$ and $R$ for which the $W_{j}$ have the same values; we write then $\rho \sim R$. Then if $\rho_{1} \sim R_{1}, \rho_{2} \sim R_{2}$, $\rho_{2} \sim R_{2}$, it is easily verified that

$$
\rho_{1} \pm \rho_{2} \sim R_{1} \pm R_{2} ; \rho_{1} \cdot \rho_{2} \sim R_{1} \cdot R_{2} ; \rho_{1}\left(\rho_{1} \pm \rho_{2}\right) \sim R_{1}\left(R_{2} \pm R_{3}\right) \text {. }
$$

Furthermore, if $\rho \rho^{\prime}=1, \rho \sim R, \rho^{\prime} \sim R^{\prime}$, then $R \cdot R^{\prime}=I$. Hence $M$ forms a field simply isomorphic with $F(\alpha)$.

THEOREM 8. The characteristic equation of any matrix $R$ of $M$ is the scome as the equation which the corresponding element $\rho$ of $F(\alpha)$ satisfies in $F$.

Proof. This follows from Theorem 7 since $\rho$ and $R$ have the same canonical form. 
We write $N(\rho)$ for the norm of any number $\rho$ of $F(\alpha)$, so that from Theorem 8:

if $R \sim \rho$, then determinant of $R=N(\rho)$;

if $R \sim \rho \neq 0$, then adjoint of $R \sim N(\rho) / \rho$ since $\operatorname{adj} R=R^{-1} \operatorname{det} R$ when $R$ is non-singular.

THEOREM 9. Any matrix of order $r$ with elements in $F$ is commutative with $M$ iff it lies in the field $M$.

Proof. If a matrix of order $r$ with elements in $F$ lies in the field $M$ then it is commutative with $M$ from Theorem 3 . If $L$ is a matrix of order $r$ over $F$ commutative with $M$ then

$$
L M=M L, L M^{2}=M^{2} L, \ldots, L M^{p-1}=M^{p-1} L .
$$

There exists a non-singular matrix $X$ which transforms $M$ into the diagonal form $M^{*}$ as in Theorem 1. By (4.4), $X^{-1} L X=L^{*}$ must also be in diagonal form. $\alpha_{r l}, \alpha_{r 2}, \ldots, \alpha_{r p}$ are the diagonal elements of $M^{*}$ from Theorem 1. Suppose that $\beta_{r 1}, \beta_{r 2}, \ldots, \beta_{r r}$ are the diagonal elements of $L^{*}$.

Now the traces of $L^{*}, M^{*} L^{*}, M^{*} L^{*}, \ldots$ are the same as the traces of $L, M L, M^{2} L, \ldots$ so that

$$
\sum_{j=1}^{r} \alpha_{r j}^{k \beta j}=I(k), k=0,1,2, \ldots, r-1,
$$

where the $I(k)$ are elements of $F$. Solving these equations for $\beta_{r^{j} j}$ $(j=1,2, \ldots, r)$, we find that as in Jarden [4],

$$
\beta_{r j}=\sum_{k=0}^{r-1} w_{k} \alpha_{r, j}^{k}, j=1,2, \ldots, r,
$$

where the $W_{k}$ are elements of $F$. Thus

$$
L^{*}=\sum_{k=0}^{r-1} W_{k} M^{*} k
$$

and 


$$
\begin{aligned}
L & =X L^{*} X^{-1} \\
& =\sum_{k=0}^{n-1} W_{k} M^{* k},
\end{aligned}
$$

as required.

THEOREM 10. Let $\left\{R_{n}\right\}$ denote the sequence of matrices defined in Theorem 6. Then a necessary and sufficient condition that $\left\{R_{n}\right\}$ should Lie in $M$ is that the sequences $\left\{w_{m, n}\right\}, m=0,1, \ldots, p-2$, be connected by the relations

$$
\text { (4.5) } \begin{aligned}
w_{m, n+j}=W_{m-1, n+j-1}+(-1)^{r-m+1}{ }_{P}, n-m^{W-1, n+j-1} & \\
& m=0,1, \ldots, n-2 ; j=0, \pm 1, \pm 2, \ldots .
\end{aligned}
$$

Proof. With the definition of matrix multiplication it is easily seen that

$$
R_{n} M=M R_{n}
$$

when and only when the relations $(4.5)$ hold. The result then follows from Theorem 9 .

Let $\left\{R_{n}\right\}$ now denote a sequence of matrices the elements of which satisfy $(4.5)$. Then

$$
R_{n}=\sum_{k=0}^{r} I(k) M_{k}^{(r)}
$$

where the $I(k), k=0,1, \ldots, r-1$, lie in $F$. By comparing the elements in the first row of both sides of this identity, we find from the initial values $M_{0}^{(r)}, M_{1}^{(r)}, \ldots, M_{p-1}^{(r)}$, that

$$
I(k)=W_{k, n},
$$

so that

$$
R_{n}=\sum_{k=0}^{r} w_{k, n} M_{k}^{(r)} .
$$

We next examine the sequences $\left\{W_{m, n}\right\}$ which are generalizations of 
the second order sequence $\left\{w_{n}\right\}$ studied in detail by Horadam [3, and elsewhere].

\section{Jacobi-Perron algorithm}

It follows from Theorem 10 that, if we extend (4.5) so that it is valid for $m=r-1$, then

$$
\frac{w_{p-j, n+1}}{W_{p-1, n+1}}=\frac{\left(w_{r-j+1, n+1} / W_{r-1, n}\right)-(-1)^{j} P_{r, i}}{\left(w_{r-2, n} / W_{r-1, n}\right)-(-1)^{1} P_{r 1}}, j=1, \ldots, r .
$$

We set

$$
a_{j-1}^{(n)}=w_{r-j, n} / w_{r-1, n} \text { and } b_{j}^{(n)}=(-1)^{j}{ }_{r j},
$$

so that if $a_{j}^{(n)}=0$ for $j \geq r$, then

$$
a_{j-1}^{(n+1)}=\left(a_{j}^{(n)}-b_{j}^{(n)}\right) /\left(a_{1}^{(n)}-\begin{array}{l}
(n) \\
1
\end{array}\right)
$$

We use the notation $E_{n-1}$ to denote the (n-1)-dimensional euclidean vector space of $(n-1)$-tuples of real numbers $(n \geq 2)$. We shall use the notation of Bernstein [2],

$$
\left(a_{1}^{(k)}, a_{2}^{(k)}, \ldots, a_{n-1}^{(k)}\right)=a^{(k)} \in E_{n-1}
$$

for $a_{i}^{(k)} \in R, i=1, \ldots, n-1$, and $k$ a non-negative rational integer.

Following Bernstein [2] we define the transformation

$$
T: E_{n-1} \rightarrow E_{n-1} \text {. }
$$

Let

$$
f\left(a^{(k)}\right)=b^{(k)}=\left(b_{1}^{(k)}, \ldots, b_{n-1}^{(k)}\right) \in E_{n-1}
$$

be any vector function on $E_{n-1}$ with values in $E_{n-1}$ such that $a_{1}^{(k)} \neq b_{1}^{(k)}$; then 


$$
a^{(k)} T=\left(a_{1}^{(k)}-b_{1}^{(k)}\right)^{-1}\left(a_{2}^{(k)}-b_{2}^{(k)}, \ldots, a_{n}^{(k)}-b_{n}^{(k)}\right) .
$$

We call $f\left(a^{(k)}\right)$ a T-function.

We further define a Jacobi-Perron Algorithm (JPA) of the vector $a^{(0)}$ as a sequence $\left\{a^{(k)}\right\}$ of vectors in $E_{n-1}$, if there exists a T-transformation of $E_{n-1}$ into $E_{n-1}$ such that for every $k$,

$$
a^{(k)} T=a^{(k+I)} \text {. }
$$

Thus

$$
\left(a^{(k)} T^{v}\right) T=a^{(k)} T^{v+1} \quad(v=1,2, \ldots)
$$

and

$$
\left(a^{(k)} T^{v}\right) T^{s}=a^{(k)} T^{v+s} \quad(s=1,2, \ldots)
$$

and

$$
a^{(k)} T^{v}=a^{(k+v)},
$$

and in particular

$$
a^{(0)} T^{k}=a^{(k)} \text {. }
$$

The JPA of $a^{(0)}$ is called periodic if there exist non-negative integers

$$
\begin{gathered}
L, \imath \geq 0 ; M, m \geq 1: T^{M+v}=T^{v} \quad(v=L, L+1, \ldots) \\
\min L=\imath, \min M=m .
\end{gathered}
$$

THEOREM 11. The JPA of $a^{(0)} \in E_{n}$ is periodic if and only if there exist integers $L \geq 0, M \geq 1:$ for $\min L=2$ and $\min M=m$,

$$
b^{(m+v)}=b^{(v)} \quad(v=0,1, \ldots) \text {. }
$$

Proof. If (5.4) holds, then $a^{(m+v)}=a^{(v)}, a^{(m+v+1)}=a^{(v+1)}$ for $v \geq \imath$, and $b^{(m+v)}=b^{(v)}$ from (5.2) for $v=\imath, 2+1, \ldots$. This proves necessity. To prove sufficiency, suppose (5.5) holds. Then from Theorem 1 of Bernstein [2], 


$$
a_{s}^{(n)}=\lim _{j \rightarrow \infty} U_{s, j}^{(r)} / U_{1, j}^{(r)} \quad(n=0,1, \ldots) .
$$

The right hand side of (5.6) is independent of $n$, so that

$$
a_{s}^{(n+m)}=\lim _{j \rightarrow \infty} U_{s, j}^{(r)} / U_{1, j}^{(r)} \quad(n=0,1, \ldots)
$$

and

$$
a_{s}^{(n)}=a_{s}^{(n+m)} \quad(n=0,1, \ldots)
$$

The complete proof of Bernstein's Theorem $I$ is too long to adapt and produce here. It involves, in analogy to the convergence of continued fractions, definitions of continued fractions and $P$-boundedness of T-functions. Indeed for $n=2, E_{n-1}$ becomes the real number space and the JPA becomes the algorithm of continued fractions.

Bernstein has also proved that if the JPA of $n-1$ numbers $(n \geq 2)$ becomes periodic, then these numbers all belong to an algebraic number field of degree $\leq n$; that is, the components of $a^{(0)}$ are all algebraic numbers of degree $\leq n$. We have thus established the following theorem:

THEOREM 12. $a^{(k)}$ belongs to an algebraic number field of degree $\leq r$.

The existence of Theorems 11 and 12 mean that Theorem 7 can also be proved by a study of the characteristic equation of the periodic JPA along the lines of Chapter 6 of Bernstein [2]. Theorem 7 can be used to establish identities for the $\left\{\begin{array}{c}(r) \\ s, n\end{array}\right\}$.

It is of interest to note in conclusion that the $r$ basic sequences satisfy the requirements of Bell [1] for a fundamental basis of order $r$ of the recurrence relation (2.1).

\section{References}

[1] E.T. Bell, "Notes on recurring series of the third order", Tôhoku Math. J. 24 (1924), 168-184. 
[2] Leon Bernstein, The Jacobi-Perron algorithm: Its theory and application (Lecture Notes in Mathematics, 207. Springer-Verlag, Berlin, Heidelberg, New York, 1971).

[3] A.F. Horadam, "Generating functions for powers of a certain generalised sequence of numbers", Duke Math. J. 32 (1965), $437-446$.

[4] Dov Jarden, Recurring sequences: A collection of papers, 2nd ed. (Riveon Lematematika, Jerusalem, 1966).

[5] Percy A. MacMahon, Combinatorial conalysis, Vol. I (Cambridge University Press, Cambridge, 1915).

[6] Morgan Ward, "The algebra of recurring series", Ann. of Math. (2) 32 (1931), 1-9.

Department of Applied Mathematics, New South Wales Institute of Technology, Broadway, New South Wales;

and

Department of Mathematics, Illinois Institute of Technology,

Chicago, Illinois, USA. 